\title{
ADSORCIÓN DEL COLORANTE AMARILLO ANARANJADO EN SOLUCIÓN ACUOSA UTILIZANDO CARBONES ACTIVADOS OBTENIDOS A PARTIR DE DESECHOS AGRÍCOLAS
}

\author{
Adolfo E. Ensuncho*1, Juana R. Robles ${ }^{1}$, José G. Carriazo ${ }^{2}$
}

\begin{abstract}
RESUMEN
Se estudió la remoción del colorante amarillo anaranjado con diferentes tipos de carbón activado, obtenidos a partir varias fuentes de desechos agrícolas. Los carbones se caracterizaron mediante espectroscopía infrarroja, difracción de rayos $\mathrm{X}$ y microscopía electrónica de barrido, obteniéndose resultados típicos de materiales carbonosos. El área superficial del carbón activo, se determinó mediante isotermas de nitrógeno. De igual manera, se evaluó la isoterma de remoción del colorante teniendo en cuenta el efecto del tiempo de contacto, el $\mathrm{pH}$, la concentración inicial y la cantidad de adsorbente. El carbón de tusa de maíz mostró mayor porcentaje remoción de colorante con respecto a los carbones cáscara de coco y cascarilla de arroz. El modelo de isoterma de Langmuir se ajusta de manera más apropiada a estos resultados. Se encontró que el modelo cinético de pseudo segundo orden describe muy bien el proceso de adsorción del colorante amarillo anaranjado con los carbones activados.

Palabras clave: Carbón activado, amarillo anaranjado, isoterma de Langmuir, modelo cinético de pseudo segundo orden, isoterma de nitrógeno.
\end{abstract}

\section{SUNSET YELLOW DYE ADSORPTION FROM AQUEOUS SOLUTIONS USING ACTIVATED CARBONS DERIVED FROM AGRICULTURAL WASTE}

\begin{abstract}
In this work was studied the adsorption of sunset yellow dye with different type of activated carbon obtained from vegetable waste. The carbons were characterized by infrared spectroscopy, X-ray diffraction and electron microscopy, obtaining typical results for carbonaceous materials. Surface areas of activated carbon were determined by nitrogen isotherms. Similarly, dye removal isotherms were evaluated taking into account, contact time effect, $\mathrm{pH}$, initial concentration and amount of adsorbent. Corn cob carbons, showed highest removal percentage with respect at the carbons of coconut husks and rice husks. Langmuir's isotherm model is adjusted more appropriately to these results. We found that, pseudo second order kinetics model describes in good agreement the adsorption of sunset yellow dye with activated carbons.
\end{abstract}

Key words: Activated carbon, sunset yellow, Langmuir's isotherm, pseudo second order kinetics model, nitrogen isotherms.

\footnotetext{
${ }^{1}$ Facultad de Ciencias Básicas, Departamento de Química, Grupo de Química Computacional, Universidad de Córdoba, Cra. 6 \#76103, Montería-Colombia. adem9abril@hotmail.com

${ }^{2}$ Facultad de Ciencias, Departamento de Química, Estado Sólido y Catálisis Ambiental (ESCA), Universidad Nacional de Colombia, Cra. 30 No. 45-03, Bogotá, Colombia.
} 


\section{INTRODUCCIÓN}

Los análisis fisicoquímicos de aguas residuales provenientes de la industria textil, cuero, cosméticos, papel, impresión, plásticos, productos farmacéuticos, alimentos, entre otros, han mostrado que contienen concentraciones significativas de colorantes, especialmente del tipo azo, lo cual ha generado una problemática ambiental ${ }^{1}$. La eliminación de estos colorantes de las aguas residuales industriales se ha convertido en uno de los principales problemas para las industrias, debido a que los colorantes son moléculas orgánicas recalcitrantes, resistentes a la digestión aeróbica, y son estables a la luz, calor y agentes oxidantes, por consiguiente difíciles de tratar'. En consecuencia, actualmente existe gran interés mundial por encontrar métodos eficientes y económicos para el tratamiento de agua. Así, por ejemplo, en la literatura científica se reporta el uso de varios métodos físicos y químicos para la remoción de colorantes presentes en aguas residuales, entre los que se encuentra la degradación microbiana aeróbica y anaeróbica, la coagulación, la oxidación química, procesos de separación de membrana, electroquímica, dilución, filtración, ósmosis reversa, entre otros. No obstante, estas metodologías resultan costosas al momento de su aplicación por lo que se requiere la búsqueda de otras alternativas que sean eficientes y económicamente atractivas.

En este sentido, el carbón activado puede ofrecer una alternativa en el tratamiento de aguas residuales, debido a que estos materiales presentan grandes áreas superficiales, elevado poder de adsorción y estructuras porosas ${ }^{3}$. En la actualidad existen investigaciones donde se utiliza el carbón activado para la remoción de contaminantes en aguas residuales. Así, Robinson y colaboradores ${ }^{4}$ reportaron que los carbones activos provenientes de residuos agrícolas, tales como cáscara de mazorca de maíz y cebada, presentaron eficientes propiedades adsorbentes de colorantes textiles en efluentes industriales. Asimismo, McKay y colaboradores ${ }^{5}$ estudiaron la adsorción del colorante azoico amarillo anaranjado a partir de soluciones acuosas sobre carbón activo, dando idea del tipo de interacciones adsorbato-adsorbente que pueden ocurrir en estos sistemas.

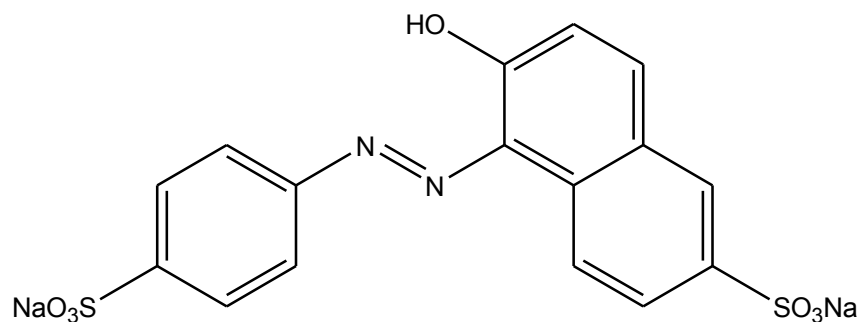

Figura 1. Estructura química de amarillo anaranjado

Por lo dicho, el objetivo que persiguió este trabajo fue obtener diferentes tipos de carbón activado a partir de desechos agrícolas, tales como cáscara de coco, cascarilla de arroz y tusa de maíz para su estudio fisicoquímico, con el fin de determinar su utilidad en la remoción del colorante azoico amarillo anaranjado (figura 1), el cual es ampliamente usado en las industrias 
de alimentos y textil. También, se evaluó la cinética de adsorción de amarillo anaranjado en los diferentes tipos de carbón activado, determinando la velocidad de remoción del colorante y el tiempo de residencia en la interface solución-adsorbente.

\section{PARTE EXPERIMENTAL}

Los carbones utilizados fueron obtenidos a partir de desechos agrícolas: cáscara de coco, cascarilla de arroz y tusa de maíz, en el Departamento de Córdoba-Colombia. Estos materiales se caracterizaron mediante espectroscopía infrarroja ${ }^{6}$, difracción de rayos X y microscopía electrónica de barrido. Las áreas superficiales de los carbones activados se determinaron a partir de las isotermas de nitrógeno. El colorante amarillo anaranjado (C.I. 15985, código FD\&C yellow $6, \lambda_{\text {máx }}=480-500 \mathrm{~nm}$ ) se obtuvo de Sigma Chemical Company.

Se preparó soluciones del colorante amarillo anaranjado midiendo una cantidad exacta del colorante disuelto en agua destilada, para preparar las soluciones madre de concentración $1 \mathrm{~g} / \mathrm{L}$; posteriormente, se preparó un grupo de soluciones de estudio con concentraciones entre $2 \mathrm{mg} / \mathrm{L}$ hasta $100 \mathrm{mg} / \mathrm{L}$. La cantidad del colorante removido se determinó mediante la búsqueda de la absorbancia en la longitud de onda característica del colorante, utilizando un Espectrofotómetro UV-Vis ThermoScientific Serie GENESYS 10S. Para determinar el efecto de la incidencia de los parámetros: tiempo de contacto $(\mathrm{t}), \mathrm{pH}$, cantidad de adsorbente $(\mathrm{m}) \mathrm{y}$ concentración inicial $\left(\mathrm{C}_{0}\right)$ en la remoción del colorante amarillo anaranjado, se llevó a cabo los experimentos a temperatura ambiente. En cada experimento se puso en contacto $10 \mathrm{~mL}$ de la solución de colorante (de concentración y $\mathrm{pH}$ deseado) con una cantidad conocida de carbón activado en un matraz de $100 \mathrm{~mL}$; esta mezcla se agitó a temperatura ambiente en un sheker a una velocidad constante de $150 \mathrm{rpm}$.

\section{Difracción de rayos $\mathrm{X}$}

\section{RESULTADOS Y DISCUSIÓN}

El análisis estructural de los tres carbones utilizados: carbón cáscara de coco (CCC), cascarilla de arroz (CCA) y tusa de maíz (CTM), se realizó por difracción de rayos X, la cual mostró perfiles típicos de materiales carbonosos ${ }^{6}$ (figura 2 ) con características bastante amorfas con señales anchas y de poca intensidad. Sólo el carbón tusa de maíz activado (CTM-A) o sin activar (CTM-SA), presentó señales intensas, propias de componentes cristalinos dentro del material. Se observó señales bastante anchas centradas en valores de espaciado interplanar d $=8,84 \AA\left(10^{\circ} 2 \Theta\right), \mathrm{d}=3,86 \AA\left(23^{\circ} 2 \Theta\right)$ y d=2,07 $\AA\left(44^{\circ} 2 \Theta\right)$; las dos últimas corresponden a los planos (002) y (101) en carbones activos6. Las señales de los planos (002) y (101) indican el apilamiento desordenado de microestructuras grafíticas o similares al grafito dentro de los materiales. Para el carbón tusa de maíz se observó una señal de difracción centrada en $10^{\circ} 2 \Theta$

$(\mathrm{d}=8,84 \AA)$; probablemente corresponda a la conformación de microestructuras laminares en los materiales carbonosos, esta señal intensa indica cristalinidad en el carbón, probablemente originada de materiales lignocelulósicos con cierto agrupamiento estructural ${ }^{7}$. 


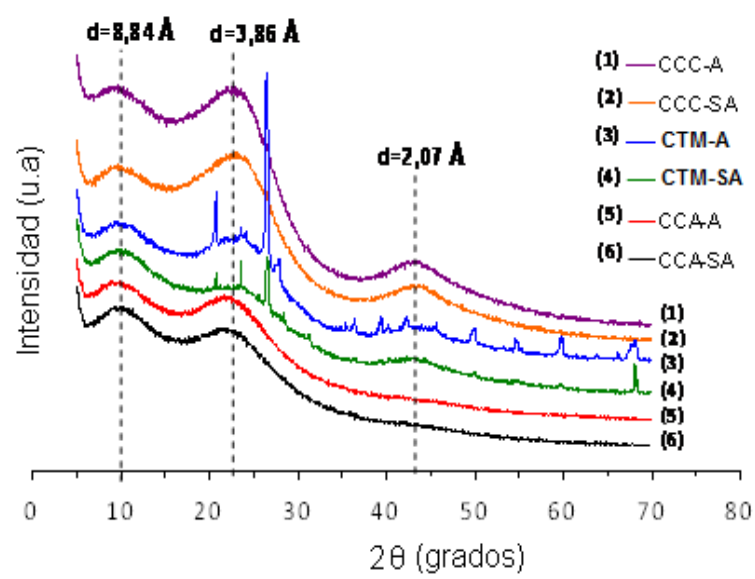

Figura 2. Perfiles de difracción de rayos X para los materiales carbonáceos activados (A) y sin activar (SA) obtenidos a partir de diferentes desechos agrícolas

\section{Espectroscopía infrarroja (IR)}

Las características químicas de los carbones activos están determinadas en gran medida por un cierto grado de heterogeneidad química de la superficie, debido a la presencia de heteroátomos, tales como: oxígeno, nitrógeno, hidrógeno, azufre y fósforo. El análisis de los diferentes grupos funcionales existentes en la superficie de cualquier sólido es importante para diferentes aplicaciones como adsorción, catálisis y reactividad del sólido, en general. Los espectros IR de los carbones obtenidos (figura 3) mostraron, bandas con vibraciones similares en todos los casos, típicas de grupos funcionales presentes en el material celulósico transformado. Dado que las muestras fueron secadas previamente a $60^{\circ} \mathrm{C}$ durante 24 horas, la señal alrededor de $3430 \mathrm{~cm}^{-1}$ puede asignarse casi en su totalidad a vibraciones de estiramiento (tensión) de los grupos $\mathrm{O}-\mathrm{H}$ formando puentes de hidrógeno ${ }^{8}$. Las señales observadas alrededor de 2920 y $2850 \mathrm{~cm}^{-1}$ se atribuyen a vibraciones simétricas y asimétricas ${ }^{8}$, de estiramiento de grupos $-\mathrm{CH}_{2}-$. El pico situado alrededor de $1560 \mathrm{~cm}^{-1}$ corresponde a las vibraciones de grupos $\mathrm{C}=\mathrm{C}$ en los anillos aromáticos de los materiales debido a la carbonización. El pico a $1613 \mathrm{~cm}^{-1}$ corresponde a grupos $\mathrm{C}=\mathrm{O}$, muy marcados en $\mathrm{CTM}$ y la señal cercana a $1100 \mathrm{~cm}^{-1}$ es asignada a vibraciones de grupos $\mathrm{C}-\mathrm{O}$ - en la misma región de $\mathrm{Si}-\mathrm{O}$; además, esta banda está acompañada de una señal de entre 400 y $500 \mathrm{~cm}^{-1}$, que se atribuye a las vibraciones de los enlaces $\mathrm{Si}-\mathrm{O}$ en sílice $\left(\mathrm{SiO}_{2}\right)^{8}$. Debe tenerse en cuenta que, la señal a $3430 \mathrm{~cm}^{-1}$ es bastante ancha y podría involucrar también vibraciones de tensión de grupos $\mathrm{O}-\mathrm{H}$ de grupos carboxílicos. La vibración en $1384 \mathrm{~cm}-1$ corresponde a flexiones de grupos $-\mathrm{CH}_{2}$. Finalmente, los picos observados en 875 y $800 \mathrm{~cm}^{-1}$ se originan debido a vibraciones de enlace $\mathrm{C}$ - $\mathrm{H}$ en los anillos aromáticos de los carbones. 


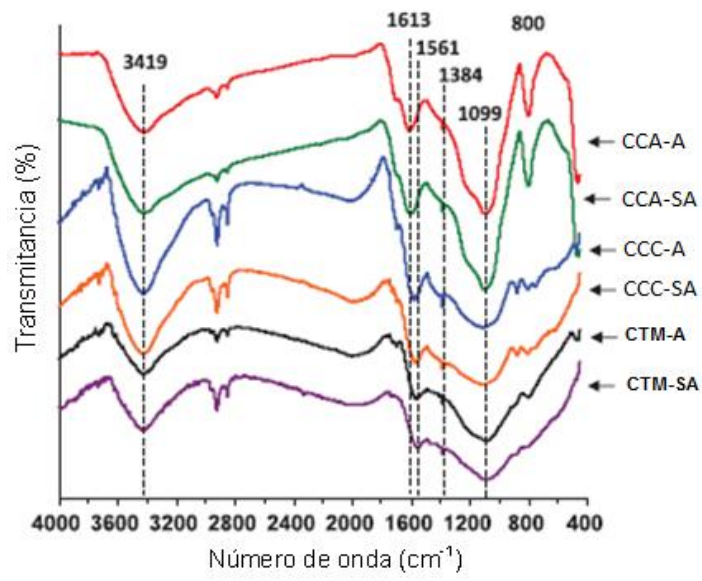

Figura 3. Espectros IR de los carbones activados y sin activar obtenidos a partir de diferentes desechos agrícolas

\section{Adsorción de nitrógeno}

En la figura 4 se muestra las isotermas de nitrógeno para los diferentes carbones preparados a partir de desechos agrícolas. Todas las isotermas de adsorción de nitrógeno fueron del tipo I, según la clasificación de la IUPAC, y son típicas de materiales microporosos. En general, todos los carbones preparados mostraron altos niveles de adsorción, especialmente el obtenido a partir de cáscara de coco (CCC). En la tabla 1, se presenta los valores de área superficial obtenidos para los materiales carbonosos preparados. De ésta, es evidente que los valores determinados por el modelo de Langmuir son más altos que los cálculos por el modelo BET, lo cual, básicamente, se debe a la naturaleza microporosa de estos materiales.

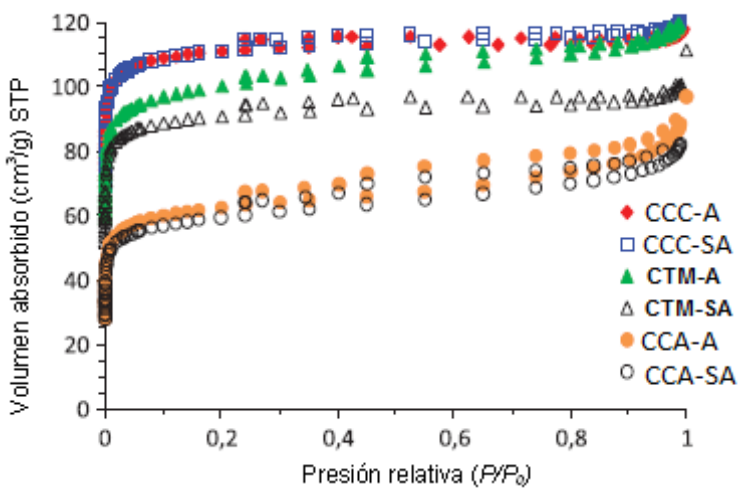

Figura 4. Isotermas de adsorción de nitrógeno a 77K de los carbones preparados a partir de los diferentes desechos agrícolas 
Los valores de área superficial de CCC son más altos que para el resto de materiales, lo cual está en buen acuerdo con los niveles de adsorción de nitrógeno descritos por las isotermas (figura 4). Además, como resultado del proceso de activación (CCC-A, CTM-A y CCA-A, respectivamente) se observa un pequeño incremento en los valores de área superficial. Adicionalmente, en la tabla 1 se presentan áreas de microporo altas, confirmando con ello la naturaleza microporosa de estos materiales. Asimismo, se calculó los volúmenes totales de poro para todos los carbones, encontrándose altos volúmenes totales de poro para $\mathrm{CCC}$ y CTM, respectivamente.

Tabla 1. Áreas superficiales, área de poro y volumen total de poro para los carbones activados (A) y sin activar (SA)

\begin{tabular}{ccccc}
\hline Material & $\begin{array}{c}\text { Área } \\
\text { BET } \\
\left(\mathrm{m}^{2} / \mathrm{g}\right)\end{array}$ & $\begin{array}{c}\text { Área } \\
\text { Langmuir } \\
\left(\mathrm{m}^{2} / \mathrm{g}\right)\end{array}$ & $\begin{array}{c}\text { Área de } \\
\text { microporo } \\
\left(\mathrm{m}^{2} / \mathrm{g}\right)\end{array}$ & $\begin{array}{c}\text { Volumen total } \\
\text { de poro } \\
\left(\mathrm{cm}^{3} / \mathrm{g}\right)\end{array}$ \\
CCC-SA & 334 & 458 & 286 & 0,1829 \\
CCC-A & 334 & 458 & 283 & 0,1853 \\
CTM-SA & 306 & 403 & 230 & 0,1894 \\
CTM-A & 274 & 373 & 228 & 0,1726 \\
CCA-SA & 192 & 245 & 135 & 0,1499 \\
CCA-A & 183 & 234 & 127 & 0,1448 \\
\hline
\end{tabular}

\section{Microscopía electrónica de barrido}

El análisis por microscopía electrónica proporciona información sobre la morfología de los sólidos, lo cual es fundamental para comprender el desempeño de los materiales en diferentes aplicaciones. La morfología variada de estos carbones preparados a partir de diferentes materiales vegetales de desechos, fue la causa de su variación en el tamaño de partícula, que en general oscila entre 20 y $500 \mu \mathrm{m}$. En todas las partículas se observó cavidades u oquedades de diferentes magnitudes, las cuales podrían contribuir al aumento del área superficial de los sólidos y cumplir con las funciones importantes en los procesos de adsorción de moléculas (figura 5).

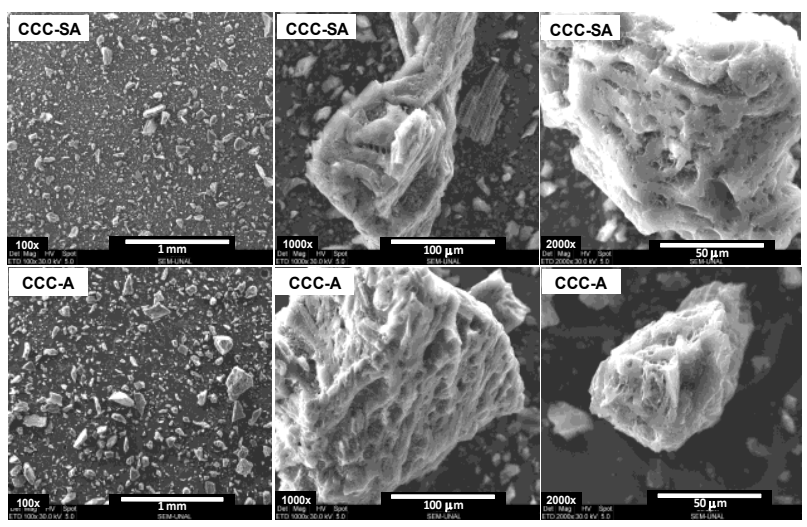

(a) 


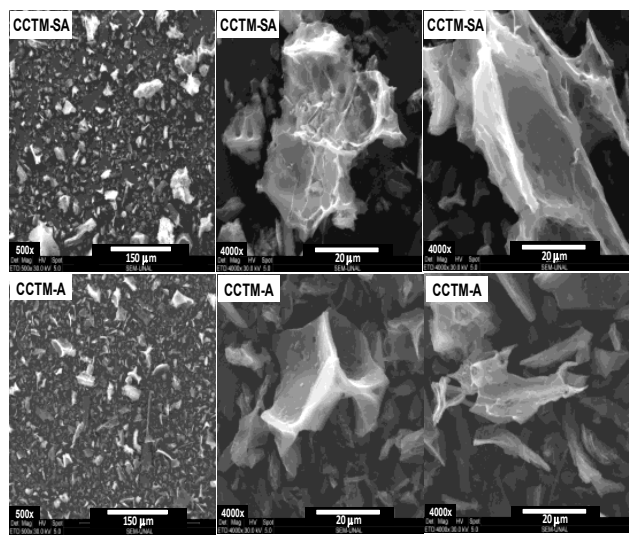

(b)

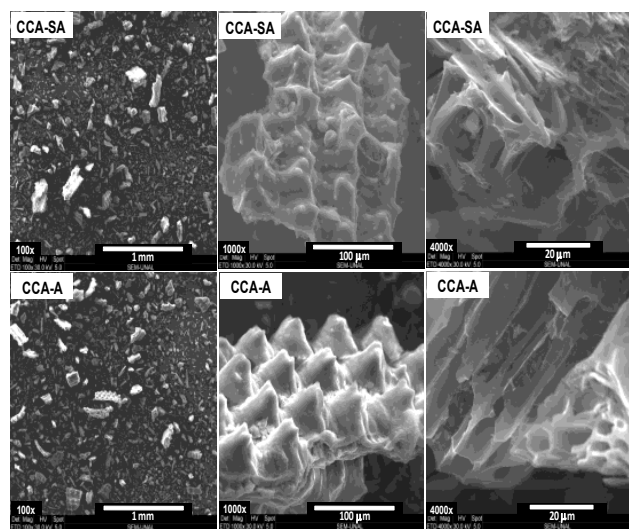

(c)

Figura 5. a) Micrografía SEM para CCC-SA y CCC-A; b) micrografía SEM para CTM-SA y CTM-A; c) micrografía SEM para CCA-SA y CCA-A

La formación de estas cavidades puede estar relacionada con la expulsión de gases y compuestos volátiles durante los procesos de calcinación, como ya se ha indicado en la literatura9 ${ }^{9}$. Las partículas de los carbones CCC, muestran una morfología más compacta que el resto de materiales. Las partículas de carbón procedentes de la carbonización de CTM poseen morfología bastante laminar en forma de hojuelas; mientras que los carbones obtenidos de cascarilla de arroz muestran superficies bastante rugosas, como ya lo habían observado otros investigadores para la cascarilla de arroz calcinada ${ }^{10}$.

\section{Adsorción del colorante amarillo anaranjado}

Para el estudio de la adsorción del colorante amarillo anaranjado sobre carbón activado, se tuvo en cuenta los siguientes parámetros: el tiempo de contacto, $\mathrm{pH}$ de la solución, cantidad de adsorbente y concentración inicial de la solución. Las condiciones para determinar los efectos de estos parámetros fueron: $\mathrm{pH}=7$, cantidad de carbón activado de $0,2 \mathrm{~g}$, una concentración de colorante amarillo anaranjado de $10 \mathrm{mg} / \mathrm{L}$ para el CCA y CCC, respectivamente, y para CTM una concentración de colorante de $70 \mathrm{mg} / \mathrm{L}$, debido a su mayor capacidad de adsorción.

\section{Efecto del tiempo de contacto}

El efecto del tiempo de contacto para la remoción del colorante amarillo anaranjado mostró una rápida adsorción por parte de los diferentes tipos de carbón en las cinco primeras horas, como se observa en la figura 5a. Posteriormente, los carbones siguen adsorbiendo, pero la relación de la cantidad de colorante adsorbido se mantiene constante. Asimismo, se observó que la máxima adsorción del colorante amarillo anaranjado se logró con CTM, en una solución de concentración 70mg/L, logrando una remoción de aproximadamente 97\% del colorante en 5 horas. Para los carbones CCC y CCA la concentración utilizada fue menor $(10 \mathrm{mg} / \mathrm{L})$, obteniéndose porcentajes de remoción de 85 y 57\%, respectivamente. Estos resultados están acordes con las estructuras morfológicas y las áreas microporosas de los carbones utilizados. 
El carbón proveniente de la tusa de maíz, mostró mayor capacidad de adsorción para este colorante, lo cual se puede atribuir a la porosidad de este material y la afinidad química entre la superficie de CTM y el colorante. Asimismo, el carbón obtenido de cáscara de coco muestra una superficie morfológica más compacta y presenta mayor área de microporo que CTM y CCA. Las partículas de los carbones obtenidos de cascarilla de arroz aparentan una morfología bastante rugosa y menor área de microporo que los otros carbones (tabla 1).

\section{Efecto del pH}

$\mathrm{El} \mathrm{pH}$ es otro factor determinante en la adsorción de colorantes en solución acuosa debido a que el $\mathrm{pH}$ afecta la estructura del colorante. Este parámetro afecta la carga del colorante y el grado de ionización del mismo con lo cual ocasiona una disminución del color en la solución. El estudio del efecto del pH en la adsorción del azocolorante amarillo anaranjado, se realizó sobre los tres tipos de carbón activado (CCC, CCA y CTM), en el rango de pH de 3 a 10. Los resultados obtenidos de la adsorción del azocolorante, se muestran en la figura 5b, e indican que a $\mathrm{pH}$ ácido ( 3 y 5), se adsorbe aproximadamente un 95\% del colorante, siendo la mayor adsorción a $\mathrm{pH} 3$; mientras que a $\mathrm{pH}$ básico, la adsorción disminuye aproximadamente en un $25 \%$. También, se observa en la figura 5b que CTM continua siendo el material carbonoso con mayor capacidad de adsorción. El colorante en solución acuosa se disocia formando el anión sulfonato del colorante y los respectivos iones sodio $\left(\mathrm{Na}^{+}\right)^{5}$. A pH ácido, la superficie del adsorbente se carga positivamente, debido a la alta concentración de iones $\mathrm{H}^{+}$en el medio; por tal motivo hay mayor atracción electrostática entre la carga positiva en la superficie del carbón y la carga negativa del anión del azocolorante. La disminución de la capacidad de adsorción a pH básico se relaciona principalmente con el incremento de la carga superficial negativa del carbón, por lo que podría producirse una repulsión electrostática con los aniones del colorante.

\section{Efecto de la concentración inicial}

Otro parámetro estudiado fue el efecto de la concentración inicial en la remoción del colorante amarillo anaranjado, por medio de carbones activos CCC, CCA y CTM, cuya importancia radica en que la determinación de este parámetro mide la resistencia de la adsorción entre la solución acuosa del colorante y la fase sólida del carbón. En la figura 6c, se muestra las concentraciones iníciales para los tres tipos de carbones activos, y se observa que la máxima adsorción corresponde a CTM, seguido por CCC y CCA, respectivamente. Los porcentajes de remoción obtenidos para el colorante amarillo anaranjado con CTM, muestra un porcentaje de remoción casi constante en todo el rango de concentraciones iniciales estudiadas. Para los carbones CCC y CCA, el máximo porcentaje de remoción se presenta a una concentración inicial de $10 \mathrm{mg} / \mathrm{L}$, a partir del cual se mantiene constante. Estos resultados indican que el cambio en la concentración afecta la tasa de saturación y tiempo de penetración en estos carbones $^{11}$. El CTM, por tener mayor capacidad de adsorción se afecta en menor grado que los otros dos carbones. En la figura 6c, es evidente que la capacidad de adsorción de los carbones CCC y CCA, es menor que en CTM lo cual se atribuye a la poca capacidad de adsorción de estos carbones debido a su estructura morfológica. 
(a)

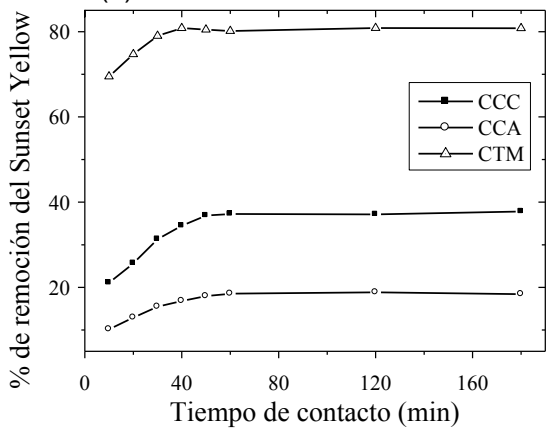

(a)

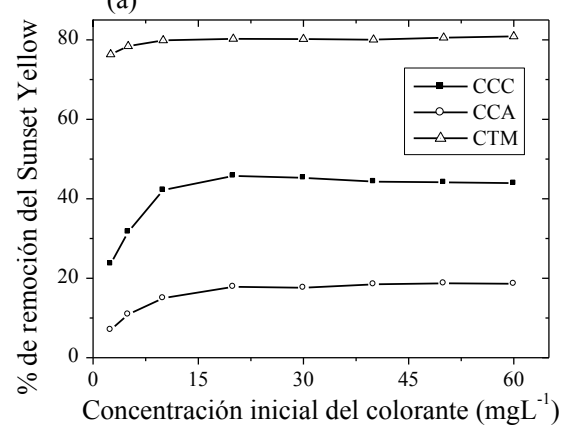

(b)

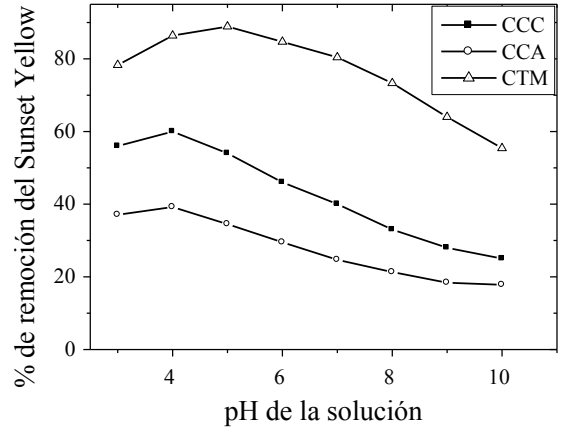

(b)

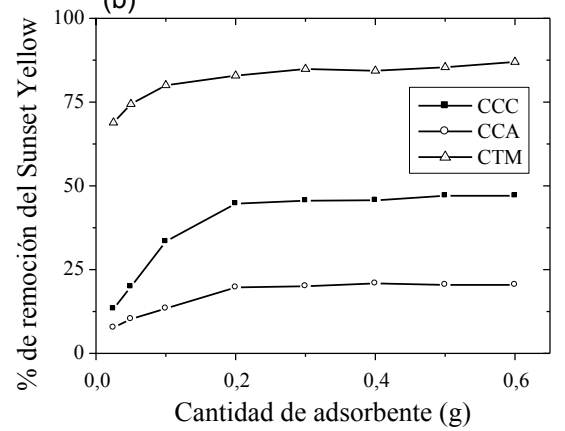

Figura 6. Isotermas de adsorción del colorante amarillo anaranjado medidos a diferentes parámetros:

(a) tiempo de contacto; (b) pH; (c) concentración inicial del colorante y (d) cantidad de adsorbente

\section{Efecto de la cantidad de carbón}

El efecto de la cantidad de adsorbente en la remoción del colorante amarillo anaranjado se estudió en un rango de 0,1 a 2,0 g de carbón. En la figura 6d, se observa un aumento gradual del porcentaje de remoción del colorante con el incremento de la cantidad de carbón, debido a que se proporciona mayor área de superficie del adsorbente y disponibilidad de más sitios de adsorción. Sin embargo, existe una cantidad límite de carbón, donde el porcentaje de remoción permanece contante. La cantidad óptima de CTM para la remoción del colorante amarillo anaranjado a partir de una solución de $70 \mathrm{mg} / \mathrm{L}$, fue de $0,3 \mathrm{~g}$. La remoción del azocolorante fue de $97 \%$. Por lo tanto, CTM remueve aproximadamente $226,33 \mathrm{mg}$ de colorante por cada gramo de carbón $(\mathrm{mg} / \mathrm{g})$. El alto porcentaje de remoción obtenido para CTM, muestra la excelente capacidad de adsorción de este material carbonoso, lo cual hace factible proponer su aplicabilidad potencial en la fabricación de filtros para la remoción de este tipo de colorantes.

\section{Isotermas de adsorción}

Las isotermas de adsorción se determinaron para la distribución del adsorbente y el adsorbato en un sistema sólido-líquido. Las isotermas de Freudlich y Langmuir son los modelos más 
utilizados para explicar el comportamiento de adsorción de estos sistemas ${ }^{9}$. Como se mostró en la tabla 1, el proceso de remoción del colorante amarillo anaranjado se ajusta al modelo Langmuir.

Para determinar el mejor modelo de isoterma de adsorción, se tomó como criterio de selección la regresión lineal comparando los coeficientes de regresión obtenidos en cada caso; siendo Langmuir el mejor modelo. La isoterma Langmuir esta descrita por la ecuación:

$$
Q_{e q}=\frac{Q_{\max } b C_{e q}}{1+b C_{e q}}
$$

Donde, $Q_{\max }$ y b son los parámetros de Langmuir relacionados a la máxima capacidad de adsorción y la energía de enlace de la adsorción, respectivamente. $Q_{e q}$, es la concentración del soluto retenido en el adsorbente en el equilibrio $(\mathrm{mg} / \mathrm{g}) . C_{e q}$, es la concentración de soluto en la solución en el equilibrio (mg/L) (figura 7).

En la tabla 2 se muestran los parámetros obtenidos cuando se describe el proceso de adsorción mediante las isotermas de Langmuir.

Tabla 2 . Parámetros de Langmuir para amarillo anaranjado

\begin{tabular}{cccc}
\hline Parámetros & CCC & CCA & CTM \\
$Q_{\max }$ & 3743,355 & 1198,609 & 6338,340 \\
$b$ & 0,024 & 0,149 & 0,015 \\
$R^{2}$ & 0,985 & 0,993 & 0,993 \\
\hline
\end{tabular}

(a) $\mathrm{CCC}$

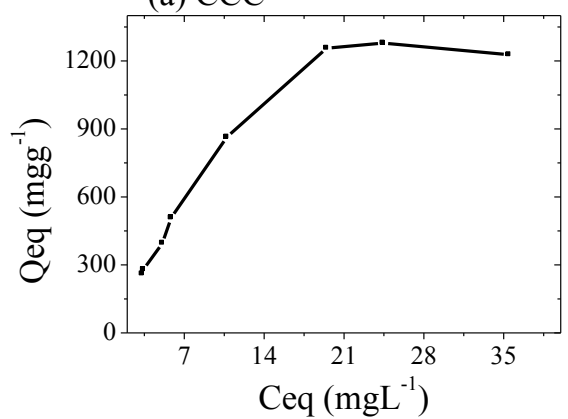

(b) CCA

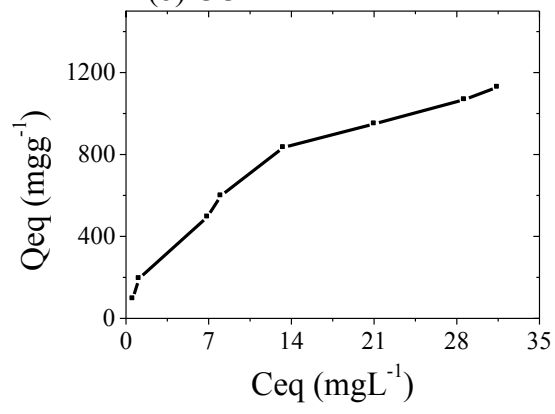




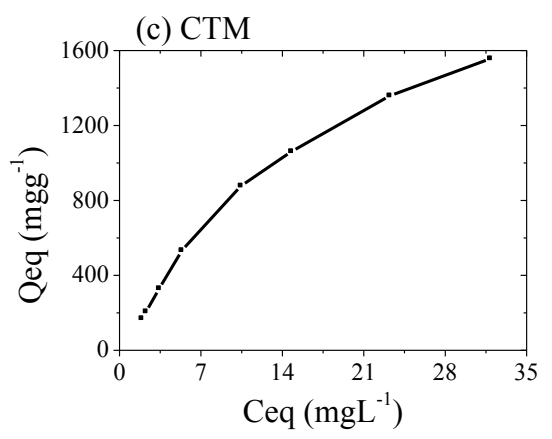

Figura 7. Isotermas de adsorción de Langmuir de amarillo anaranjado para: (a) CCC, (b) CCA y (c) CTM, respectivamente.

\section{Cinética de la adsorción}

El estudio cinético del proceso de adsorción permitió determinar la velocidad de adsorción del colorante amarillo anaranjado en la fase acuosa. Se probó varios modelos cinéticos para describir el proceso de acuerdo con los diferentes parámetros medidos en las isotermas de adsorción. De acuerdo con los datos cinéticos presentados en la tabla 3, se observa que la cinética adsorción se ajusta a un modelo cinético de pseudo-segundo orden, ya que, presenta mayores valores de $\mathrm{R}^{2}$. El modelo cinético de pseudo-segundo orden se expresa como:

$$
\frac{t}{q_{t}}=\frac{1}{K_{2} q_{e}^{2}}+\frac{1}{q_{e}} t
$$

Donde: $k_{2}$ es la constante de velocidad de adsorción, qe es la cantidad de colorante adsorbida en el equilibrio $(\mathrm{mg} / \mathrm{g})$ y qt es la cantidad de colorante adsorbido en el tiempo $\mathrm{t}(\mathrm{mg} / \mathrm{g})$. La capacidad de equilibrio de adsorción $\left(q_{e}\right)$ y la constante de velocidad de segundo orden $\left(k_{2}\right)$ $(\mathrm{g} / \mathrm{mgmin})$ puede determinarse experimentalmente a partir de la pendiente $\mathrm{y}$ la intersección en el gráfico de $t / q$ versus $t$.

En la tabla 3 se presentan las constantes de velocidad, capacidad de equilibrio y $\mathrm{R}^{2}$ para los diferentes carbones investigados.

Tabla 3. Parámetros cinéticos de los modelos de pseudo-primer y segundo orden para amarillo anaranjado con diferentes carbones activados.

\begin{tabular}{ccrcccc}
\hline Tipo de & \multicolumn{3}{c}{ Modelo cinético pseudo-primer orden } & \multicolumn{3}{c}{ Modelo cinético pseudo-segundo orden } \\
carbón & $\mathrm{K}_{1}\left(\mathrm{~min}^{-1}\right)$ & $\mathrm{q}_{\mathrm{e}}\left(\mathrm{mgg}^{-1}\right)^{*}$ & $\mathrm{R}^{2}$ & $\mathrm{~K}_{2}\left(\mathrm{gmg}^{-1} \mathrm{~min}^{-1}\right)$ & $\mathrm{q}_{\mathrm{e}}\left(\mathrm{mgg}^{-1}\right)$ & $\mathrm{R}^{2}$ \\
CCC & 0,389 & 1241,90 & 0.412 & $6,570 \times 10^{-3}$ & 162,601 & 0,997 \\
CCA & 0,935 & 3040,90 & 0,406 & $5,970 \times 10^{-4}$ & 102,354 & 0,996 \\
CTM & 0,935 & 3040,90 & 0,116 & $1,780 \times 10^{-3}$ & 373,134 & 0,998 \\
\hline
\end{tabular}


En la figura 8 se muestra las gráficas de $t / q$ vs. $t$ para los diferentes carbones investigados. Los resultados de la cinética de adsorción obtenidos en este trabajo están en buen acuerdo con reportes experimentales previos para carbones activados, donde se ha mostrado que la cinética se ajusta a un modelo de pseudo-segundo orden ${ }^{12}$.
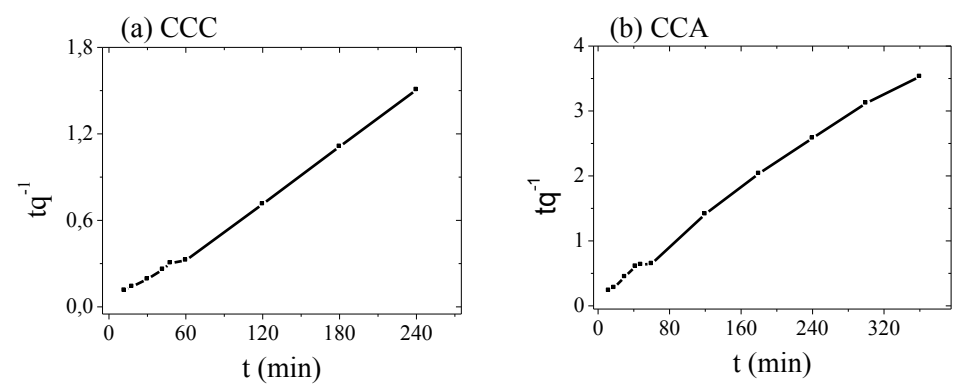

(c) CTM

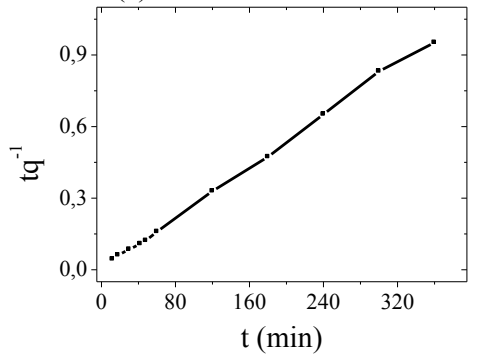

Figura 8. Gráficas de log (qe-q) versus t, modelo cinético pseudo-segundo orden para el amarillo anaranjado con diferentes carbones activos. (a) Carbón cáscara de coco, (b) Carbón cascarilla de arroz y (c) Carbón tusa de maíz

\section{CONCLUSIONES}

Los resultados de la caracterización de los carbones mostraron que se obtuvo carbones activos con áreas superficiales de $458 \mathrm{~m}^{2} / \mathrm{g}$ para carbón cáscara de coco, $373 \mathrm{~m}^{2} / \mathrm{g}$ para el carbón tusa de maíz y $234 \mathrm{~m}^{2} / \mathrm{g}$ para carbón cascarilla de arroz y unas áreas de microporo de $283 \mathrm{~m}^{2} / \mathrm{g}$, $228 \mathrm{~m}^{2} / \mathrm{g}$ y $127 \mathrm{~m}^{2} / \mathrm{g}$, respectivamente.

Se encontró que el carbón obtenido de tusa de maíz (CTM) tiene una alta capacidad de adsorción del azocolorante amarillo anaranjado como lo indicaron los porcentajes de remoción. El carbón obtenido de cáscara de coco presentó niveles de adsorción buenos, debido a que presenta una típica adsorción física donde prima su mayor área de microporo y el carbón de cascarilla de arroz no presentó buenas características como material adsorbente para este colorante, ya que presenta menor área de microporo.

Para los tres tipos de carbones utilizados en la remoción del colorante amarillo anaranjado, el modelo cinético que mejor explicó el proceso de adsorción fue el de pseudo-segundo 
orden, y las isotermas de adsorción se ajustaron mejor al modelo de Langmuir. La adsorción del azocolorante con carbón activado mostró una sensibilidad a la concentración inicial, el tiempo de contacto, el pH de la solución y la cantidad de adsorbente.

\section{AGRADECIMIENTO}

Los autores de este artículo muestran su agradecimiento al Centro de Investigaciones Universitarias (CIUC), de la Universidad de Córdoba, por el apoyo económico brindado (proyecto: código 1.2.08.109 y numeral FCB-03-09).

\section{BIBLIOGRAFÍA}

1. M.S. Chiou, P.Y. Ho, H.Y. Li. Dyes Pigments; 2004; 60: 69.

2. A. Kamal. J. Hazard. Mater; 2009; 165: 52.

3. K.A. Wojciech et. al. Chem. Eng. J; 2013; 228: 824.

4. T. Robinson. Water Res; 2002; 36: 2824.

5. G. McKay, G. Ramprasad, P. Pratapa Mowl. Water Air Soil Poll; 1986; 29: 273.

6. J.G. Zhao, F.Y. Li, C.Q., Jin. Solid State Commun; 2009; 149: 818.

7. P. Barpanda, G. Fanchini, G.G., Amatucci. Carbon; 2011; 49: 2538.

8. L. Huang, Y. Sun, W. Wang, Q. Yue, T. Yang. Chem. Eng. J; 2011; 171: 1446.

9. Y. Sun, et. al. Chem. Eng. J; 2012; 181: 790.

10. M. Asadullah et. al. J. Hazard. Mater; 2010; 174: 437.

11. $\quad$ L. Wang et. al. Desalination; 2010; 254: 68.

12. M. Dogan, M.H. Karaoğlu, M. Alkan, J. Hazard. Mater; 2009; 165: 1142. 